\title{
Impact of fabrics from transgenic flax on cultures of skin cells
}

\author{
Kazimierz Gąsiorowski ${ }^{1, A, C, E, F}$, Tomasz Gębarowski ${ }^{1, B-D}$, Helena Moreira' ${ }^{1, B, C}$, Anna Kulma ${ }^{2, E, F}$, Michał Szatkowski ${ }^{2, E}$, Jan Szopa ${ }^{2, A, E}$ \\ ${ }^{1}$ Department of Basic Medical Sciences, Faculty of Pharmacy, Wroclaw Medical University, Poland \\ ${ }^{2}$ Department of Genetic Biochemistry, Faculty of Biotechnology, University of Wroclaw, Poland \\ A - research concept and design; $B$ - collection and/or assembly of data; $C$ - data analysis and interpretation; \\ $D$ - writing the article; $E$ - critical revision of the article; $F$ - final approval of the article
}

Address for correspondence

Kazimierz Gąsiorowski

E-mail: kazimierz.gasiorowski@umed.wroc.pl

\section{Funding sources}

Applied Research Programme of the National Centre

for Research and Development - PBS1/A9/17/2012

Conflict of interest

None declared

Received on September 30, 2016

Reviewed on February 13, 2018

Accepted on June 25, 2018

Published online on January 17, 2019

\begin{abstract}
Background. The development of a new type of wound dressing material that can support skin regeneration is an important challenge to improve treatment of chronic, non-healing wounds.

Objectives. The objective of this study was to compare the impact of flax fabrics from transgenic plants overexpressing phenolic acids and flavonoids (W92) and polyhydroxybutyrate (M48), as well as fabric from non-transgenic plant (Nike) on cultures of human skin cells.

Material and methods. Flax fabric pieces as well as water extracts from the fabrics were co-cultured with human skin cells: keratinocytes, fibroblasts, dermal microvascular endothelial cells, and with monocytoid cell line (THP1) for 48 h. Cell viability and proliferation were assessed with the sulforhodamine B colorimetric assay. Intracellular reactive oxygen species (ROS) was estimated with the $2^{\prime} 7$ dichlorodihydrofluorescein diacetate (DCFH-DA) oxidation method. Endothelial cell migration was measured with the scratch assay. The results were compared with the multi-criteria analysis (MCA) procedure.
\end{abstract}

Results. Tested flax fabrics released flavonoids and polyhydroxybutyrate to cell culture media, as it was determined by means of the high performance liquid chromatography (HPLC) method. Fabrics from transgenic plants W92 and M48 promoted proliferation of keratinocytes and fibroblasts. Water extracts from flax fabric diminished the proliferation of monocytoid cells, decreased oxidative burst in activated THP1 cells and accelerated the velocity of dermal microvascular cell migration. The MCA proved that the sum of beneficial effects estimated in human skin cell cultures was higher (by 47\% and by 34\% with W92 and M48, respectively) than that of non-transgenic flax fabric (Nike).

Conclusions. The W92 and M48 fabrics should be further studied as candidates for elaboration of new types of bandages, able to improve skin wound healing.

Key words: flax fabric, skin cell cultures, flax biotechnology, transgenic flax fabrics

Cite as

Gąsiorowski K, Gębarowski T, Moreira H, Kulma A,

Szatkowski M, Szopa J. Impact of fabrics from

transgenic flax on cultures of skin cells. Adv Clin Exp Med.

2019;28(4):431-438. doi:10.17219/acem/92563

DOI

10.17219/acem/92563

Copyright

Copyright by Author(s)

This is an article distributed under the terms of the

Creative Commons Attribution Non-Commercial License

(http://creativecommons.org/licenses/by-nc-nd/4.0/) 


\section{Introduction}

Flax (Linum usitatissimum), an annual plant primarily cultivated for industrial purposes, was recently genetically modified in order to enhance wound healing properties of the fibers. ${ }^{1-3}$

These new types of flax have been genetically modified in order to provide stable overexpression of enzymes for polyhydroxybutyrate (PHB) synthesis, ${ }^{1}$ and enzymes of phenylpropanoid pathway. ${ }^{3}$ Three-gene construct with cDNA encoding $\beta$-ketothiolase ( $p h b A$ ), acetoacetyl-CoA reductase $(p h b B)$ and PHB synthase $(p h b C)$ generated a new flax type called $M$ type composite fiber. It contains a new kind of cellulose-polyhydroxybutyrate polymer with components chemically bound via hydrogen and ester bonds. Those fibers were previously found to promote the proliferation rate of cultured fibroblasts. ${ }^{4}$ The W type flax plants were generated by the introduction of 3 genes from the phenylpropanoid pathway (chalcone synthase, chalcone isomerase and dihydroflavonol reductase). ${ }^{3} \mathrm{New}$ flax fibers overexpressing these genes accumulate components like phenolic acids and flavonoids with antioxidant activity. ${ }^{2}$

Previously, we reported the impact of wound dressings made of fibers from W92 plants on healing chronic skin ulcers. ${ }^{5,6}$ A 4-week application of bandages composed of the W92 flax fabric led to a marked reduction of wound exudates and significantly decreased wound size in $55 \%$ of patients. Beneficial effect of flax fabrics impact on a healing wound needs further studies in cultures of cells engaged in skin regeneration and repair. In this paper, we describe the impact of fabrics from fibers of M48 and W92 transgenic flax plants and from non-transgenic Nike flax on proliferation in vitro of human primary skin cells: keratinocytes and fibroblasts. Flax fabrics were cut into small pieces of $0.25 \mathrm{~cm}^{2}, 0.5 \mathrm{~cm}^{2}$ and $1 \mathrm{~cm}^{2}$, sterilized and added to cell cultures for $48 \mathrm{~h}$. Also, the proliferation of non-adherent human monocytoid cells (THP1 cell line) and a content of intracellular reactive oxygen species (ROS) in those cells as well as a velocity of endothelial cell migration in vitro were established in cultures with the water extracts from fabrics. Results obtained with the tests were compared by means of the multi-criteria analysis (MCA) procedure. ${ }^{7,8}$ The MCA calculations allowed us to compare the effects of tested linen fabrics in order to choose the most favorable material as a candidate for a new type of bandage that can improve wound healing.

\section{Material and methods}

\section{Plant material}

Genetically modified flax were obtained according to the procedures described previously ${ }^{3,9}$; the M48 flax expressed genes for PHB synthesis: $\beta$-ketothiolase $(p h b A)$, acetoacetyl-CoA reductase $(p h b B)$ and PHB synthase (phb C), and the W92 overexpressed genes from the phenylpropanoid pathway: chalcone synthase, chalcone isomerase and dihydroflavonol reductase. Plants were grown in a field in the 2010 vegetative season. After 4 months of field growth, the plants were harvested and the flax fiber was prepared via the standard dew retting process, which has previously been described. ${ }^{10}$ The fabrics were sterilized by autoclaving at $120^{\circ} \mathrm{C}$ for $20 \mathrm{~min}$.

\section{Reagents and cell culture media}

KBM-Gold medium (Keratinocyte Cell Basal Medium), medium supplements, Dulbecco's modified Eagle's medium (DMEM), and fetal bovine serum (FBS) were purchased from Lonza (Verviers, Belgium). Endothelial Cell Growth Medium MV and its supplements were obtained from PromoCell (Heidelberg, Germany). The solution of antibiotics (10,000 U/mL penicillin and $10,000 \mu \mathrm{g} / \mathrm{mL}$ streptomycin) containing $29.2 \mathrm{mg} / \mathrm{mL} \mathrm{L}$-glutamine was purchased from HyClone Laboratories Inc. (Logan, USA) and the Trypsin EDTA solution from Lonza. Sulforhodamine B (SRB), phorbol 12-myristate 13-acetate (PMA) and Trizma-base were purchased from Sigma-Aldrich (St. Louis, USA). Phosphate buffered saline (PBS) and $0.4 \%$ of trypan blue solution were obtained from POCH SA (Gliwice, Poland).

\section{Estimation of phenolic components released from the flax fabrics to cell culture medium}

In order to determine the amount of phenolic components released from the flax fabrics to cell culture medium, $10 \mathrm{~cm}^{2}$ fragments of the tested fabrics were incubated in $10 \mathrm{~mL}$ of medium (DMEM, without FBS) for $48 \mathrm{~h}$ at $37^{\circ} \mathrm{C}$. After incubation, the solution was adjusted to $\mathrm{pH} 3$ and extracted 3 times with ethyl acetate. The ethyl acetate fraction was dried under a vacuum, resuspended in $1 \mathrm{~mL}$ of methanol and analyzed using liquid chromatography in Ultra Performance Liquid Chromatography (UPLC; Waters Corp., Milford, USA).

\section{Cells and cell growth conditions}

Three types of primary skin cells from adult donors: normal human epidermal keratinocytes (NHEK), normal human dermal fibroblasts (NHDF) and human dermal microvascular endothelial cells (HDMEC) were purchased from PromoCell (Heidelberg, Germany). Established monocytoid cell line (human acute monocyte leukemia, THP1) was obtained from the Laboratory for Therapeutic Innovation, Faculty of Pharmacy, University of Strasbourg, France.

Cell pellets were suspended with PBS, counted and inspected under a microscope for cell viability with the standard trypan blue exclusion test. Then, the cells were plated on 24-well plastic culture plates and sterile pieces $\left(0.25 \mathrm{~cm}^{2}, 0.5 \mathrm{~cm}^{2}\right.$ and $\left.1.0 \mathrm{~cm}^{2}\right)$ of flax fabrics were placed in each culture-well of adherent cells, or respective 
volume of aqueous extract from the flax fabric was added to the cultures of non-adherent cells. Cultures were incubated in a $\mathrm{CO}_{2}$-incubator at $37^{\circ} \mathrm{C}$ for $48 \mathrm{~h}$.

\section{Determination of the cell density/cell proliferation}

Cell density/cell proliferation was determined with the sulforhodamine B (SRB)-colorimetric assay. ${ }^{11}$ Sulforhodamine absorbance was estimated at $490 \mathrm{~nm}$ with the Victor 2 microplate reader (PerkinElmer, USA).

\section{Evaluation of the intracellular free radical level}

Free radical content in THP-1 cells was estimated with the dichlorodihydrofluorescein diacetate (DCFH-DA) oxidation method. ${ }^{12}$ The standard activator of free radical generation (PMA, $200 \mathrm{nM}$ ) was added for $2 \mathrm{~h}$ to selected cultures. Fluorescence of intracellular, oxidized DCFH-DA was inspected at $525 \mathrm{~nm}$ (FL1) with the CyFlow Space cytometer (Partec, Germany), equipped with a $488 \mathrm{~nm}$ argon laser lamp.

\section{Estimation of endothelial cell migration}

The impact of the aqueous extract from the flax fabric on migration of human dermal microvascular endothelial cells was analyzed on plastic Petri dishes using the scratch assay in vitro. ${ }^{13}$ An analysis of cell migration distances and calculations of the velocity of cell migration $(\mu \mathrm{m} / \mathrm{h})$ were carried out with AxioVision software (Carl Zeiss, Germany).

\section{Statistical analysis}

The statistical significance of the results (mean \pm standard deviation - SD; $\mathrm{n}=5$ ) was assessed with the paired t-test with Microsoft Excel 2010 software (Microsoft Corp., Redmond, USA) and significant results were marked on histogram columns with asterisks (*p $<0.05$, ** $\left.\mathrm{p}<0.001,{ }^{* * *} \mathrm{p}<10^{-4}\right)$.
The multi-criterial analysis (MCA) procedure was calculated according to literature data ${ }^{7,8}$ with own modifications. In brief, the results obtained with 6 in vitro tests with $1.0 \mathrm{~cm}^{2}$ area of the flax fabrics pieces or equivalent dose of water extract from fabrics were compared to the relative control cultures $\left(E / E_{0}\right)$ and the statistical distances between the expected (exp.) and observed (obs.) results were calculated with the standard formula: $b=(\text { exp. }- \text { obs. })^{2} /$ exp. The expected values in each test were assumed as the most favorable results estimated for 3 tested flax fabrics. Results of the calculation were expressed as $1 / \mathrm{b}$ ratios and then multiplied by the indices of importance. The indices of importance were assumed for the results of each test, given their potentially beneficial role in skin wound healing. Finally, the results of the MCA procedure of compared fabrics were obtained according to the following equation: $M=\Sigma 1 / b$.

\section{Results}

Tested flax fabric released detectable amount of phenolic compounds to aqueous media, as revealed by HPLC analysis.

Table 1 shows that, despite differences in the content and release of individual identified phenolic compounds, the total amount of phenolic compounds released into aqueous solutions after $48 \mathrm{~h}$ of incubation of the tested flax fabrics in the PBS solution was more than twice as high for W92 as compared to the Nike flax. In the case of M48 fabric, during the 48-hour incubation, 30\% less phenolic compounds were released into the aqueous solution than from the Nike fabric, although the total antioxidant activity of this extract was comparatively strong as the Nike extract.

Additionally, the M48 fabric released polyhydroxybutyrate, which degrades to D,L- $\beta$-hydroxybutyrate in contact with body fluids (data not shown). ${ }^{1}$ Similar results were obtained with the flax fabrics incubated for $48 \mathrm{~h}$ in cell culture media (without FBS) - all identified phenolic compounds were released to the cell culture media.

Table 1. Content of phenolic compounds [ng] released from $1 \mathrm{~cm}^{2}$ of fabric after $48 \mathrm{~h}$ incubation in phosphate-buffered saline solution (PBS) and antioxidant activity of the water extracts

\begin{tabular}{|c|c|c|c|c|c|c|}
\hline \multirow{2}{*}{ Estimated phenolic compound } & \multicolumn{2}{|c|}{ Nike } & \multicolumn{2}{|c|}{ W92 } & \multicolumn{2}{|c|}{ M48 } \\
\hline & ng & $\%$ of total & ng & $\%$ of total & ng & $\%$ of total \\
\hline 3,4 dihydroxybenzoic acid & 18.34 & 25.06 & 23.19 & 27.27 & 10.68 & 11.23 \\
\hline 4-hydroxybenozoic acid & 10.31 & 6.73 & 22.71 & 10.97 & 9.06 & 3.67 \\
\hline 4-hydroxy-3-methoxybenzoic acid (vanilic acid) & 21.82 & 7.76 & 10.37 & 4.82 & 12.57 & 4.29 \\
\hline Vanilin & 14.50 & 1.90 & 16.08 & 1.73 & 11.13 & 0.59 \\
\hline Ferulic acid & 4.40 & 5.40 & 5.88 & 4.28 & 4.50 & 3.22 \\
\hline Vitexin & 8.35 & 7.69 & 6.97 & 4.77 & 4.15 & 2.86 \\
\hline Total & 77.82 & 5.30 & 163.02 & 10.20 & 56.29 & 3.10 \\
\hline Antioxidative properties (\% of oxidation inhibition) & \multicolumn{2}{|c|}{42} & \multicolumn{2}{|c|}{48} & \multicolumn{2}{|c|}{43} \\
\hline
\end{tabular}



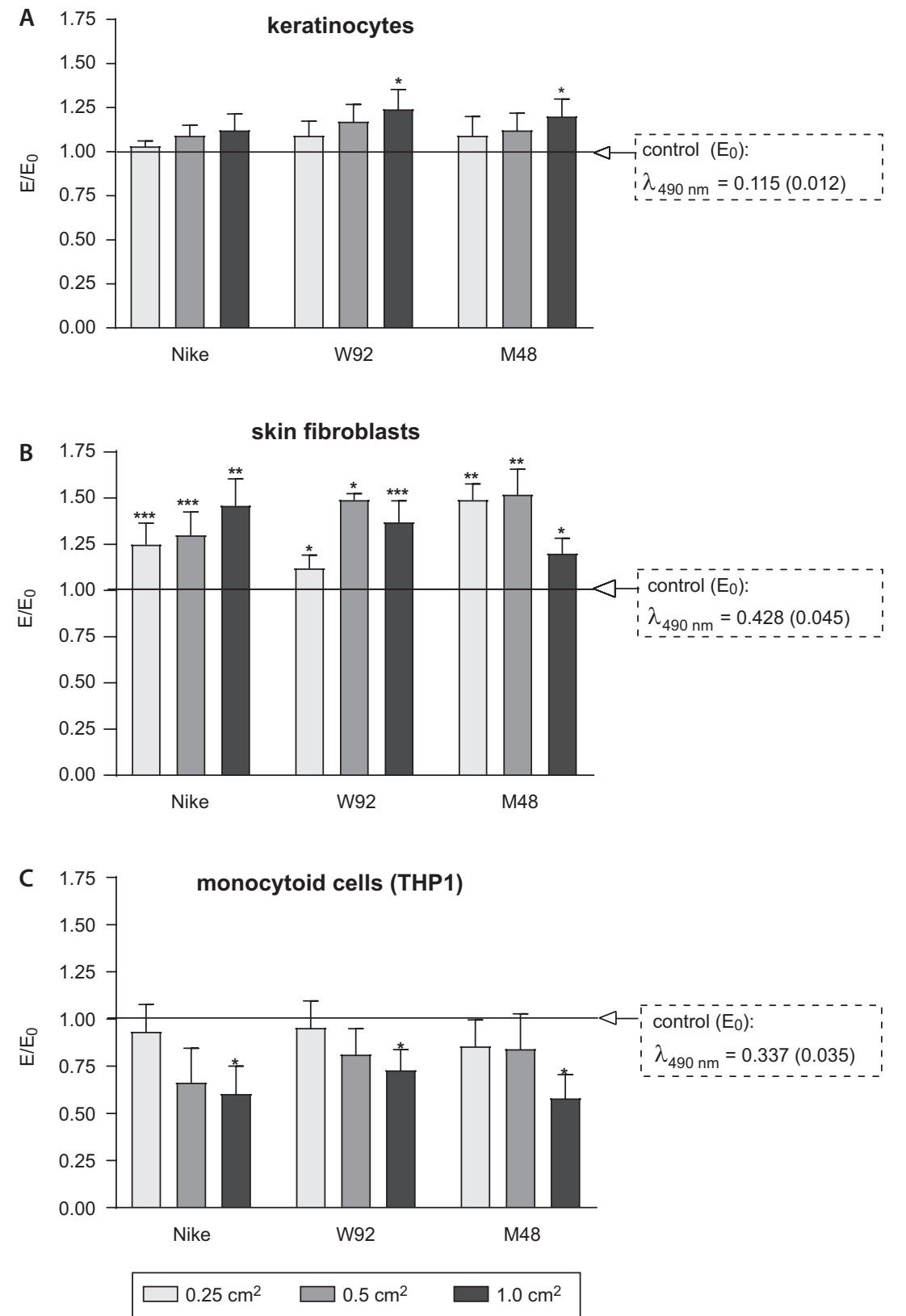

surface area of flax fabric pieces or corresponding doses of flax fibers extracts added to cell cultures

Incubation of keratinocytes and skin fibroblasts in the presence of the tested flax fabrics pieces lead to marked increase of cell proliferation, measured with the SRB test. On the other hand, monocytoid cells (THP1) cultured in the presence of fabrics water extract exhibited significant decrease of cell proliferation.

Impact of flax fabric pieces (keratinocytes and fibroblasts) and water extract from fabrics (THP1) on skin cell proliferation was shown in Fig. 1. The results were compared to relative control cultures (without flax fabric, $\left.E_{0}=1\right)$ and presented as $E / E_{0}$ ratio in histograms in Fig. 1A-C.

Figure $1 \mathrm{~A}$ shows a moderate increase of cell number of human keratinocyte cultures after 48-hour incubation with the tested flax fabrics. The effect was directly
Fig. 1. Proliferation of keratinocytes, skin fibroblasts and monocytoid cells (THP1) estimated with the sulforhodamine B (SRB) test after culture of cells for $48 \mathrm{~h}$ in the presence of tested flax fabrics pieces (A and B) or in the presence of water extract from equivalent area of fabric pieces (C). The results (E) were compared to those obtained in the relative control cultures (without flax fabrics; $E_{0}=1.0$ ) and shown as $E / E_{0}$ ratios. Control $E_{0}$ values $(\lambda 490 \mathrm{~nm}$ ) were given as mean (standard deviation - SD; $n=5$ ). Statistical significance of the results obtained in tested and in control cultures was calculated with t-test $\left({ }^{*} p<0.05\right.$; ** $p<0.001$; *** $p<10^{-4}$ ) 
A

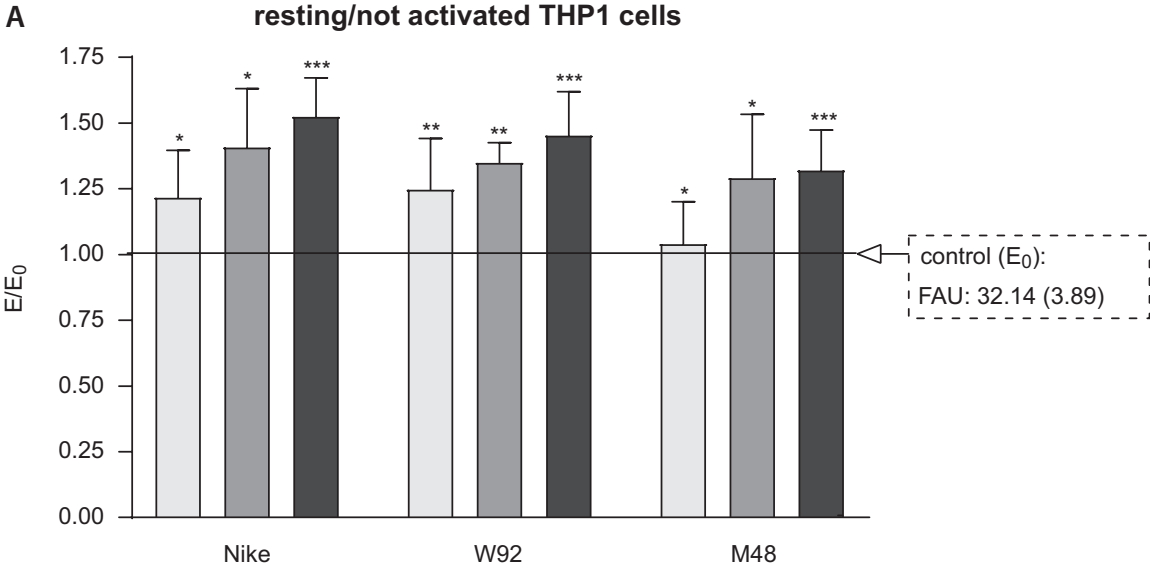

Fig. 2. Intracellular ROS contents in monocytoid cells (THP1) estimated with the dichlorodihydrofluorescein diacetate (DCFH-DA) oxidation test after 48-hour incubation of THP1 cells in the presence of water extracts from the tested flax fabric pieces. THP1 cells were not activated/ /resting cells (A) or activated with phorbol 12-myristate 13-acetate (PMA; 200 nM, 2 h) (B). The results (E) were compared to those obtained in the relative control cultures (without flax fabrics extracts; $E_{0}=1.0$ ) and shown as $E / E_{0}$ ratios. Control $E_{0}$ values (fluorescence arbitrary units - FAU) were shown as mean (standard deviation - SD; $n=5$ ). Statistical significance of the results obtained in tested and in control cultures was calculated with t-test $\left({ }^{*} p<0.05\right.$; ${ }^{* *} p<0.001$; *** $p<10^{-4}$ )

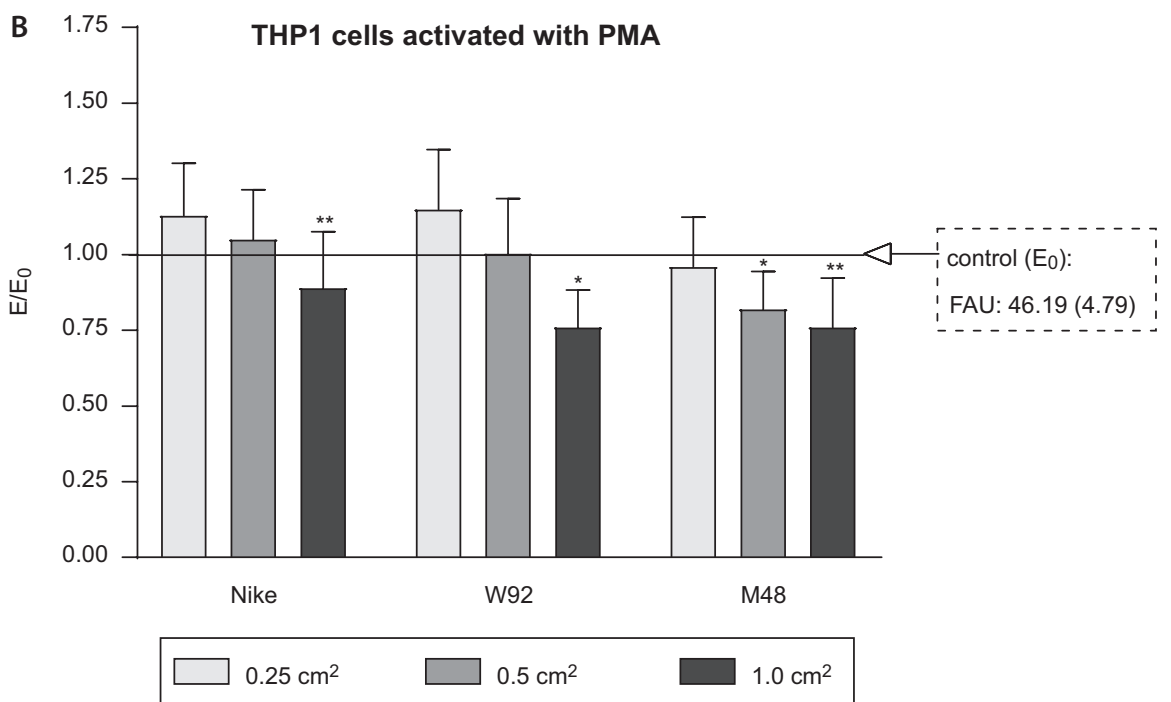

present in cell culture, and in the presence of $1 \mathrm{~cm}^{2}$ pieces of the fabric the cell proliferation was even by $46 \%$ higher than in control culture.

Figure $1 \mathrm{C}$ illustrates the influence of flax fabric water extracts on proliferation of human monocytoid cell line (THP1). Tested flax fabrics markedly decreased the proliferation of THP1; decrease by $42 \%$ (M48), by $40 \%$ (Nike) and by $28 \%$ (W92) were estimated for $1 \mathrm{~cm}^{2}-$ area of the pieces of the fabric, compared to the control culture.

The intracellular contents of ROS in the THP1 cells cultured for $48 \mathrm{~h}$ with the extracts from tested flax fabrics (resting/not activated cells) and in THP1 cells stimulated to oxidative burst by the treatment with phorbol ester (PMA, $200 \mathrm{nM}$ for $2 \mathrm{~h}$ ) were estimated with the standard DCFH-DA oxidation test. The results were compared to those obtained in relative control cultures $\left(\mathrm{E}_{0}\right.$; cells cultured without the presence of flax fabric extracts) and shown in Fig. 2 as E/ $\mathrm{E}_{0}$ ratios.

In resting THP1 cells, not activated to oxidative burst, the aqueous extracts from the 3 types of flax fabric enhanced ROS production in THP1 cells, as was shown in Fig. 2A. The increase was the highest at the dose of 1.0 $\mathrm{cm}^{2}$ of extracted flax fabric, by more than $50 \%$ in the case of the Nike, by $45 \%$ for the W92, and by $32 \%$ for the M48 fabric when compared to the level of ROS in the control culture (without fabric extract; $\mathrm{E}_{0}=1.0$ ).

In THP1 cells activated to oxidative burst with PMA, lower doses of water extracts from the tested flax fabrics $\left(0.25 \mathrm{~cm}^{2}\right.$ of fabric extracted) caused a small increase (by about $10 \%$ in the case of the Nike and W92 extracts) of intracellular ROS, whereas higher doses of extracts $\left(1.0 \mathrm{~cm}^{2}\right.$ fabric extracted) led to a marked decrease of the ROS contents in THP1 cells by about $10 \%$ (Nike) and by almost $25 \%$ (W92 and M48), as is presented in Fig. 2B.

Mean velocity of HDMEC migration $(\mu \mathrm{m} / \mathrm{h})$ in the presence of aqueous extracts from $1.0 \mathrm{~cm}^{2}$ pieces of the flax fabrics and in the control cell culture (without tested extracts) is presented in Fig. 3. Flax fabric extracts differed markedly in their impact on endothelial cell migration. The cell migration velocity was increased by $33 \%$ in the presence of the extract from M48 fabric and by $20 \%$ with the extract from W92 fabric, whereas the extract from non-transgenic Nike fabric caused a decrease by $50 \%$ of cell migration velocity when compared to the control culture (without tested extracts).

The results obtained with 6 tests were evaluated in order to choose the most advantageous flax fabric type 
Table 2. Multi-criterial analysis (MCA) of 3 flax fabric in the aspect of their potential enhancement of skin wound healing

\begin{tabular}{|l|c|c|c|c|}
\multicolumn{1}{|c|}{ Rating and ranking criteria } & \multicolumn{2}{c|}{ Tested flax fabric } \\
\hline \multicolumn{1}{|c|}{ Tests } & expected result & $\begin{array}{c}\text { index } \\
\text { of importance }\end{array}$ & Nike \\
\hline Proliferation of keratinocytes (NHEK) & increase & 0.25 & 6.280 & 26.880 \\
\hline Proliferation of fibroblasts (NHDF) & increase & 0.15 & 9.145 & 3.212 \\
\hline Proliferation of monocytoid cells (THP1) & decrease & 0.20 & 4.194 & 1.220 \\
\hline ROS in resting THP1 cells & increase & 0.05 & 3.820 & 1.730 \\
\hline ROS in activated THP1 cells & decrease & 0.20 & 1.390 & 0.711 \\
\hline Migration of endothelial cells (HDMEC) & increase & 0.15 & 0.199 & 4.540 \\
\hline Total MCA (sum of 1-6) & & 1.00 & 2.112 \\
\hline
\end{tabular}

NHEK - normal human epidermal keratinocytes; NHDF - normal human dermal fibroblasts; ROS - reactive oxygen species; HDMEC - human dermal microvascular endothelial cells.

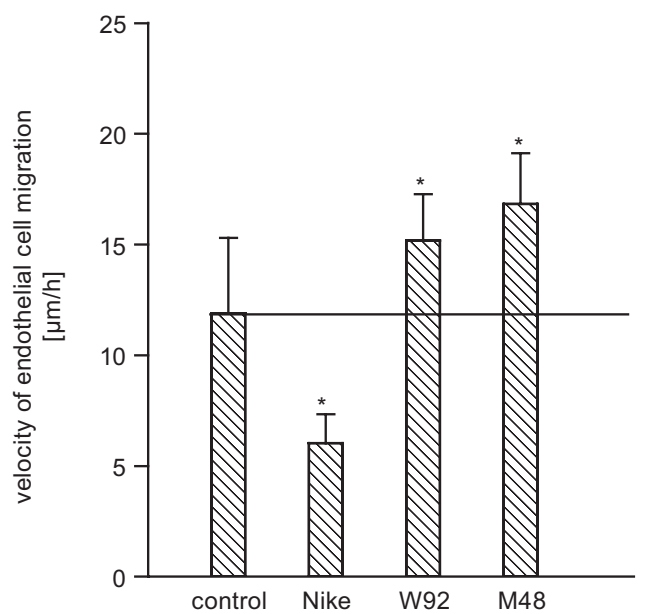

Fig. 3. Velocity of human endothelial cell migration in vitro in the presence of water extracts from the tested flax fabrics $\left(1.0 \mathrm{~cm}^{2}\right.$ of flax fabric pieces extracted with water). Significance of the results obtained with flax fabric extracts and those obtained in control cultures (without the extracts) were calculated with t-test $\left({ }^{*} p<0.05\right)$

for elaboration of new skin wound healing bandage. The results of the MCA are given in Table 2.

The overall beneficial effect on skin cell cultures (the MCA index) was higher by about $47 \%$ in the case of W92 fabric and by 34\% in the case of M48 than that of non-transgenic Nike flax fabric. The analysis proved that W92 flax fabric exhibited the most favorable overall effect on skin cell cultures, which could be important in the promotion of wound healing.

\section{Discussion}

An increasing number of patients suffering from chronic, non-healing skin wounds are a serious challenge for modern medicine. Thus, the search for new type of dressing materials able to promote skin wound healing becomes an urgent need. The aim of our study was to find a flax fabric for a new type wound dressings, whose effects on the skin cells cultures could suggest possible improvement in skin wound healing. A favorable dressing material should release active compounds to the moist environment of a wound, which could promote healing the skin injuries. The tested flax fabrics released a detectable amount of polyphenolics and polyhydroxybutyrate to water solutions and to cell culture media; also, they differed in the capacity of the release - the compounds from the W92 fabric were the most easily released. The reason for this is unknown yet, but earlier experiments suggested that the cellulose polymers in W92 fiber were less tightly bound than those in M48 and this might affect extraction efficiency. ${ }^{2}$ Probably the more dense fiber structure of the M48 fabric $^{2}$ results in a slower release of phenolic compounds from this fabric to the aqueous solution in a 48-hour incubation procedure.

We compared the impact of 2 types of transgenic flax fabrics (W92 and M48) and non-transgenic fabric (Nike) on the proliferation, intracellular ROS and migration of human skin cells. The tested primary cell cultures of keratinocytes, fibroblasts and HDMEC were taken from adult human donors, since chronic skin wounds afflict primarily elderly people. ${ }^{14}$ In chronic wounds, the ability of keratinocytes and fibroblasts to proliferate and to release cytokines and growth factors is markedly reduced. ${ }^{15,16}$ Therefore, new medicinal materials able to promote wound healing should stimulate the proliferation of these cells as a crucial element of connective tissue repair and epithelial wound closure. The tested flax fabrics enhanced the proliferation of keratinocytes and fibroblasts in cell culture, and the effect of the W92 fabric was the strongest.

It was documented that cells at the non-healing edges of chronic wounds reveal a decreased ability to migrate and differentiate, ${ }^{17}$ whereas endothelial cell migration is an indispensable condition of angiogenesis and vasculogenesis, prerequisite for effective wound healing. ${ }^{18}$ Therefore, we checked the impact of the flax fabrics on the in vitro endothelial cell migration, In our study, the extracts from the engineered flax fabrics (W92 and M48) enhanced cell migration, while the extract from non-transgenic flax fabric (Nike) lowered migration of endothelial cells as compared to the control culture (without fabric extract). 
Chronic inflammatory reactions are considered a regular feature of non-healing skin wounds, being induced and sustained by monocytes/macrophages, which proliferate and release ROS inside a wound. ${ }^{19}$ Thus, it was suggested that treatment focusing on the reduction of monocytoid cell proliferation could decrease local chronic inflammation within a wound and enhance the healing processes in chronic wounds. We estimated the proliferation potential and free radical release from established human monocytoid cell line THP1 - the cell line which is often used in studying the mechanisms of monocyte/macrophage participation in inflammatory reactions. ${ }^{20}$

Incubation of the THP1 cells for $48 \mathrm{~h}$ in the presence of the aqueous extract from flax fabric decreased proliferation of monocytoid cells. We expected that also in vivo these extracts could decrease monocyte cell burden in human skin wounds, attenuating local inflammatory reactions. The influence of the tested extracts from flax fabrics on ROS generation in resting (not activated) THP1 cells leads to significant increase of the ROS levels, especially in the case of the non-transgenic fabric, when compared to the control cell culture (without fabric extract). However, the intracellular level of ROS was markedly decreased in THP1 cells cultured with the tested extracts and subsequently, stimulated to ROS production with PMA, a strong activator of free radical generating enzymes. ${ }^{21}$ The decreasing effect depended on the dose of extracts and was the most significant at the highest extract dose, especially with W92 and M48 extracts.

Although an excess of free radicals is deleterious and apparently should be attenuated to prevent cellular damage and death, a normal, physiological level of free radicals is a necessary component of many intracellular signal transduction pathways, thereby playing a vital role in the cellular stress response and in tissue repair. ${ }^{22}$ For instance, free radicals are essential regulators of endothelial cell functions during new vessel formation in wound healing $^{18,23}$ and, apparently, are crucial for the host defense against microbial infections. ${ }^{22}$ Indeed, a fine-tuning modulator of ROS is required in chronic wound treatment, able to decrease free radical excess without affecting the basic pool of ROS needed for normal physiological processes and intracellular signaling and necessary for resistance against bacterial infection. According to our results, we can conclude that the M48 and W92 flax fabrics are promising modulators of free radical level, able to moderately increase the basic pool of ROS, and to decrease the free radical excess in cells activated to ROS generation.

The results of our in vitro study revealed that the tested flax fabrics from transgenic plants as well as the extracts obtained from the fabrics improved cell activities which could be important for wound healing. A comparison of the results obtained with 6 tests using the MCA shows that the potentially beneficial influence on skin wound healing could be markedly stronger with fabrics from transgenic flax - by $47 \%$
(W92) and by 34\% (M48) when compared to non-transgenic flax fabric (Nike). The W92 and M48 flax fabrics seem to be a promising material for the development of a new type of dressings, able to improve skin wounds healing.

\section{ORCID iDs}

Kazimierz Gąsiorowski (i) https://orcid.org/0000-0003-4467-3488 Tomasz Gębarowski (D) https://orcid.org/0000-0002-8742-0790 Helena Moreira (1) https://orcid.org/0000-0002-8084-3686 Anna Kulma (D) https://orcid.org/0000-0002-5073-901X Michał Szatkowski (D) https://orcid.org/0000-0002-0437-4734 Jan Szopa (i) https://orcid.org/0000-0003-0621-597X

\section{References}

1. Szopa J, Wrobel-Kwiatkowska M, Kulma A, et al. Chemical composition and molecular structure of fibers from transgenic flax producing polyhydroxybutyrate, and mechanical properties and platelet aggregation of composite materials containing these fibers. Compos Sci Technol. 2009;69(14):2438-2446.

2. Zuk M, Kulma A, Dyminsk Lamalice L, Le Boeuf F, Huot J. Endothelial cell migration during angiogenesis. Circ Res. 2007;100(6):782-794.

3. Lorenc-Kukula K, Amarowicz R, Oszmianski J, et al. Pleiotropic effect of phenolic compounds content increases in transgenic flax plant. J Agric Food Chem . 2005;53(9):3685-3692.

4. Kunert-Keil C, Gredes T, Meyer A, Wrobel-Kwiatkowska M, Dominiak M, Gedrange T. The survival and proliferation of fibroblasts on biocomposites containing genetically modified flax fibers: An in vitro study. Ann Anat. 2012;194(6):513-517.

5. Skorkowska-Telichowska K, Zuk M, Kulma A, et al. New dressing materials derived from transgenic flax products to treat long-standing venous ulcers: A pilot study. Wound Repair Regen. 2010;18(2):168-179.

6. Skorkowska-Telichowska K, Czemplik M, Kulma A, Szopa J. The local treatment and available dressings designed for chronic wounds. J Am Acad Dermatol. 2013;68(4):e117-e126.

7. Beck $B$, Iversen $P$, Shashegy $A$. Combining information for quantitative decision-making in drug development. JMCDA. 2014;21:139-151.

8. Nutt DJ, King LA, Phillips LD. Drug harms in the UK: A multicriteria decision analysis. Lancet. 2010;376(9752):1558-1565.

9. Wróbel M, Zebrowski J, Szopa J. Polyhydroxybutyrate synthesis in transgenic flax. J Biotechnol. 2004;107(1):41-54.

10. Wróbel-Kwiatkowska M, Zebrowski J, Starzycki M, Oszmianski J, Szopa J. Engineering of PHB synthesis causes improved elastic properties of flax fibers. Biotechnol Prog. 2007;23(1):269-277.

11. Vichai V, Kirtikara K. Sulforhodamine B colorimetric assay for cytotoxicity screening. Nat Protoc. 2006;1(3):1112-1116.

12. Eruslanov E, Kusmartsev S. Identification of ROS using oxidized DCFDA and flow-cytometry. Methods Mol Biol. 2010;594:57-72.

13. Liang C-C, Park AY, Guan J-L. In vitro scratch assay: A convenient and inexpensive method for analysis of cell migration in vitro. Nat Protoc. 2007;2(2):329-333.

14. Cheng S, Chen GQ, Leski M, Zou B, Wang Y, Wu Q. The effect of D,Lbeta-hydroxybutyric acid on cell death and proliferation in $L 929$ cells. Biomaterials. 2006;27(20):3758-3765.

15. Telgenhoff D, Shroot B. Cellular senescence mechanisms in chronic wound healing. Cell Death Differ. 2005;12(7):695-698.

16. Seah CC, Phillips TJ, Howard CE, et al. Chronic wound fluid suppresses proliferation of dermal fibroblasts through a Ras-mediated signaling pathway. J Invest Dermatol. 2005;124(2):466-474.

17. Usui ML, Mansbridge JN, Carter WG, Fujita M, Olerud JE. Keratinocyte migration, proliferation, and differentiation in chronic ulcers from patients with diabetes and normal wounds. J Histochem Cytochem. 2008;56(7):687-696.

18. Pendyala S, Gorshkova IA, Usatyuk PV, et al. Role of Nox4 and Nox2 in hyperoxia-induced reactive oxygen species generation and migration of human lung endothelial cells. Antioxid Redox Signal. 2009;11(4):747-764.

19. Clanchy FIL, Holloway AC, Lari R, Cameron PU, Hamilton JA. Detection and properties of the human proliferative monocyte subpopulation. J Leukoc Biol. 2006;79(4):757-766. 
20. Winkler C, Ueberall F, Fuchs D. In vitro testing for anti inflammatory properties of compounds. Clin Chem. 2006;52(6):1201-1202.

21. Goel G, Makkar HPS, Francis G, Becker K. Phorbol esters: Structure, biological activity, and toxicity in animals. Int J Toxicol. 2007;26(4):279-288.
22. Jiang F, Zhang Y, Dusting GJ. NADPH oxidase-mediated redox signaling: Roles in cellular stress response, stress tolerance, and tissue repair. Pharmacol Rev. 2011;63(1):218-242.

23. Lamalice L, Le Boeuf $F$, Huot J. Endothelial cell migration during angiogenesis. Circ Res. 2007;100(6):782-794. 